\title{
Discrete-Time Dynamical Maximum Power Tracking Control for a Vertical Axis Water Turbine with Retractable Blades
}

\author{
Zhaoyong Mao, Qian Liu, and Rongxin Cui \\ School of Marine Science and Technology, Northwestern Polytechnical University, Xian 710072, China \\ Correspondence should be addressed to Zhaoyong Mao; maozhaoyong@nwpu.edu.cn
}

Received 28 November 2015; Revised 3 February 2016; Accepted 22 February 2016

Academic Editor: Driss Boutat

Copyright (C) 2016 Zhaoyong Mao et al. This is an open access article distributed under the Creative Commons Attribution License, which permits unrestricted use, distribution, and reproduction in any medium, provided the original work is properly cited.

This paper addresses the power generation control system of a new drag-type Vertical Axis Turbine with several retractable blades. The returning blades can be entirely hidden in the drum, and negative torques can then be considerably reduced as the drum shields the blades. Thus, the power efficiency increases. Regarding the control, a Linear Quadratic Tracking (LQT) optimal control algorithm for Maximum Power Point Tracking (MPPT) is proposed to ensure that the wave energy conversion system can operate highly effectively under fluctuating conditions and that the tracking process accelerates over time. Two-dimensional Computational Fluid Dynamics (CFD) simulations are performed to obtain the maximum power points of the turbine's output. To plot the tip speed ratio curve, the least squares method is employed. The efficacy of the steady and dynamic performance of the control strategy was verified using Matlab/Simulink software. These validation results show that the proposed system can compensate for power fluctuations and is effective in terms of power regulation.

\section{Introduction}

With the development of economy and society, requirement for energy resource increases continuously. As a kind of ocean energy, ocean current energy is an abundant and renewable energy. For example, in UK, the government has the target of electricity demand from renewable sources, $15 \%$ by 2015 and $20 \%$ by 2020 . In Scotland, the goal for 2020 is $50 \%$ [1]. Oceans cover $75 \%$ of the world's surface, so, the ocean energy is a global resource. Using ocean energy for electric power generation is an area of research interest, and, currently, most of our work focuses on the cost-effective utilization of this energy to ensure quality and reliability in electricity delivery. Over the last two decades, increasing varieties of wave energy systems have been designed, and different concepts have been developed and tested [2-4].

Characterized by their rotational axis orientation with regard to the water flow direction, water turbines can be classified as Horizontal Axis Turbines (HATs) and Vertical Axis Turbines (VATs). In addition, depending on the type of driving force that rotates the rotor, VATs can be classified into two categories: lift-type and drag-type. Darrieus turbines are the most efficient type of lift-type turbine; these turbines use airfoils to create the lift needed to maintain rotation. Darrieus turbines have been studied extensively, using CFD and experimental methods to optimize their performance $[5,6]$. In comparison to lift-type turbines, drag-type turbines have better versatility and include Savonius turbines, Zephyr turbines, and Banki turbines. Savonius turbines have a number of advantages and have been studied experimentally and numerically regarding the effects of various design parameters, such as the rotor aspect ratio, the overlap, the number of buckets, the rotor endplates, and the influence of bucket stacking $[7,8]$.

In a drag-type VAT, such as the Savonius turbines, the blades will experience negative torque during the returning half cycle, which limits their power efficiency [9-11]. This paper describes a new drag-type VAT that consists of several retractable blades mounted in a drum. The axial position of each blade is controlled to achieve a high output performance. To reduce the negative torque in the returning half cycle and eventually increase the net driving torque, we adopt a blade control mechanism comprising a drum, a synchronous belt, and several links and shafts to reduce the negative torque 
on the returning blade. The blades are mounted inside the drum and controlled independently by the blade control mechanism. In the advancing half cycle, the blades are pushed out of the drum, generating positive torque to drive the rotor, whereas in the returning half cycle, the blades are pulled into the drum, reducing the negative torque [12].

For the control part, the system's main control objective is to maximize the power efficiency, which requires the turbine tip speed ratio to be maintained at its optimum value regardless of wave variations. Nevertheless, control does not always aim to capture as much energy as possible. In fact, the optimal turbine speed varies from one wave speed to another. This paper's contribution lies in its employment of an effective MPPT control and optimal LQT control for varying operating conditions. Optimal LQT control is one of the most successful control algorithms and is widely used in handling multivariables and constraints [13-17]. The designed control discipline is based on a mathematic model of a controlled object with the prescribed limit to acquire the optimal performance index [18-20]. According to previous research on PID control, the conventional control algorithm gives good results at infinite steady state; the only difficulty occurs when the reference trajectory is fluctuating [21, 22]. This paper builds on the ideas presented in [23]. The performance of a controller using optimal LQT control and PID will be analyzed and discussed in the simulation section.

\section{Ocean Energy Conversion System}

Water turbines can be characterized by their rotational axis orientation with regard to the water flow direction. VATs, which are also known as cross-flow water turbines, rotate around an axis perpendicular to the current. VATs are less efficient than their horizontal counterparts, but they can operate regardless of the flow direction and are more suitable for small-scale, distributed power generation. VATs' water energy conversion system is composed of a hub, blades, a nacelle, a gearbox, a generator, and a power regulator controller (see Figure 1). The device's operating principle is similar to that of wind turbines. Each wave washes over the streamlined blades at some speed and some angle, leading to the rotation of the shaft. The hydrodynamic kinetic energy is then converted into mechanical energy. The gearbox transforms the low-speed, high-torque mechanical energy and passes it to the generator, regulating the power and simultaneously exporting the load. Therefore, these turbines are also called "underwater windmills."

2.1. Description of the VAT. The rotational period of a VAT can be divided into the advancing half cycle and the returning half cycle because the blades generate positive torque in the former and negative torque in the latter. The VAT described in this paper is quite different from previous designs. Here, we adopted a blade control mechanism consisting of a drum, a synchronous belt, and several links and shafts to reduce the negative torque on the returning blade (see Figure 2). The blades are mounted inside the drum and controlled independently by the blade control mechanism. In the advancing

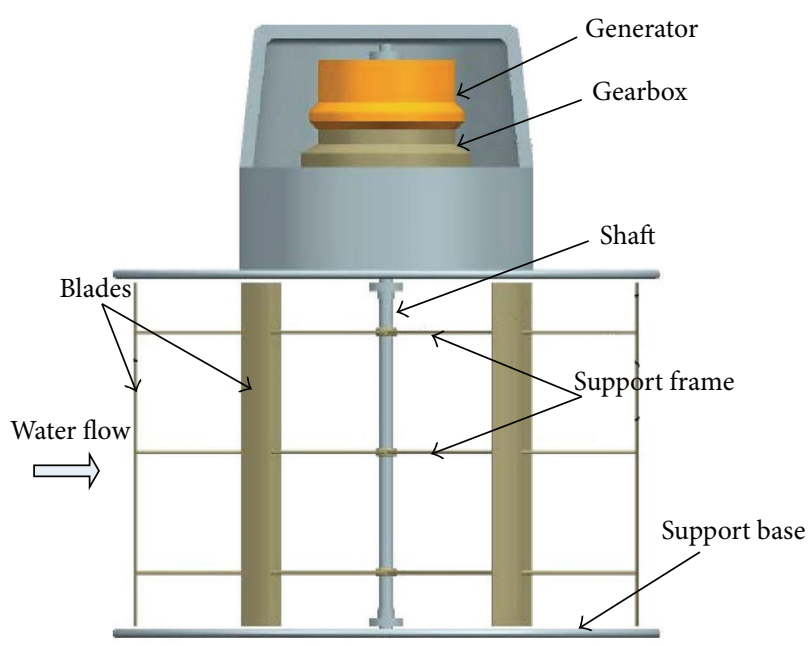

FIGURE 1: VAT water energy conversion system.

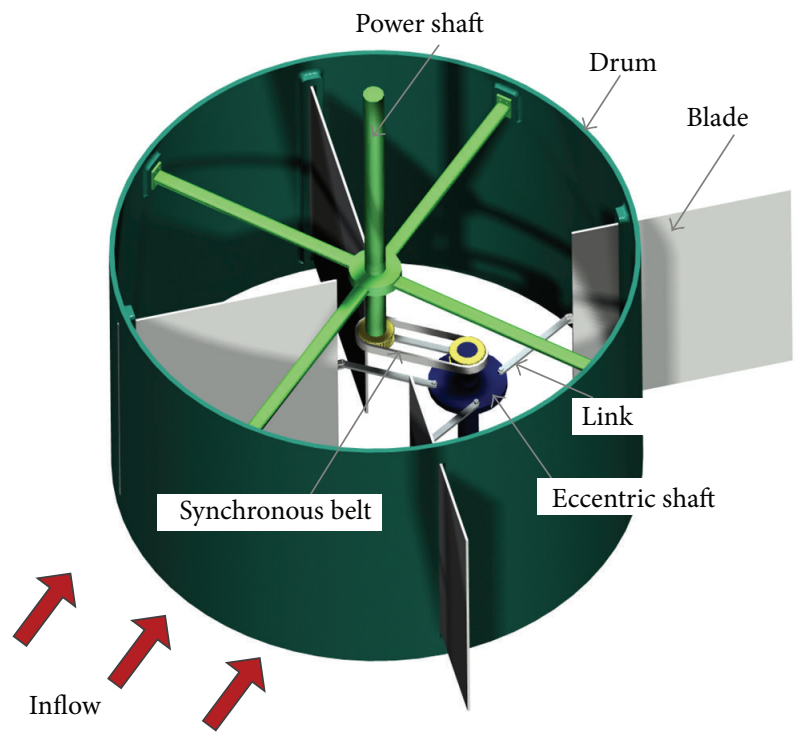

FIGURE 2: VAT with retractable blades.

half cycle, the blades are pushed out of the drum, generating positive torque to drive the rotor, whereas in the returning half cycle, the blades are pulled into the drum, reducing the negative torque.

The turbine works as follows: the blades are first pushed out of the drum by the links as the turbine rotates, generating positive torque to drive the rotor. Then, after fully extending, the blades retract to the inside of the drum, allowing water to flow past the turbine with minimum resistance. The resultant torque drives the turbine and produces power.

Figure 3 presents a two-dimensional schematic of the turbine mechanism and illustrates some important parameters: $U$ is the velocity of the incident flow water, $R$ is the diameter of the drum, $c$ is the eccentricity between the power shaft and the eccentric shaft, $r$ is the radius of the eccentric disc, $l$ is the length of the link, $L$ is the length of the blade, and $\theta$ is 
TABLE 1: VAT characteristics.

\begin{tabular}{lc}
\hline Rating & Value \\
\hline Diameter of the drum & $R=0.5 \mathrm{~m}$ \\
Eccentricity between the power and eccentric shaft & $c=0.225$ \\
Radius of the eccentric disc & $r=0.05$ \\
Length of the link & $l=0.225$ \\
Length of the blade & $L=0.45$ \\
\hline
\end{tabular}

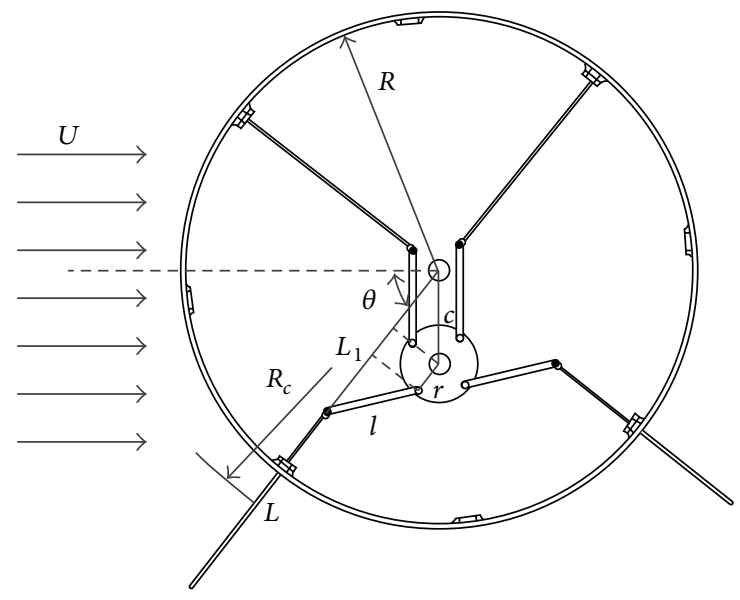

FIgURE 3: Schematic of the turbine mechanism.

the rotational angle, which is used to describe the rotation of the blade relative to the incident flow. $L_{1}$, the distance between the outer edge of the blade and the drum axis, can be calculated as

$$
L_{1}=c \sin \theta+\sqrt{l^{2}-c^{2} \cos ^{2} \theta}+r+L .
$$

In the advancing half cycle $(0 \leq \theta<\pi), L_{1}$ is maximized when $\theta=\pi / 2$ for water impacting the blade perpendicularly, and the blade achieves a relatively high driving torque. In the returning half cycle $(\pi \leq \theta<2 \pi), L_{1}$ remains $r+L$ (constant). This finding confirms that the blades are entirely hidden in the drum and only rotate around the power shaft. Negative torques can thus be considerably reduced because the drum shields the blades. VAT.

Table 1 shows the main geometric characteristics of the

2.2. Kinetic Characteristics of the System. According to Betz theory [24], the hydrodynamic power $P_{a}$ captured by the water turbine is given by

$$
P_{a}=\frac{1}{2} \pi \rho R^{2} C_{p}(\lambda) v^{3} .
$$

In this function, $C_{p}$ represents the power conversion efficiency, which depends on the tip speed ratio $\lambda$ and the blade pitch angle $\beta$ in a pitch-controlled water turbine. It is a nonlinear function representing the ability of the water energy conversion system to maximize energy capture. $\lambda$ is the ratio of the tip speed of the turbine blades to the wave speed and is defined as

$$
\lambda=\frac{R \omega_{r}}{v} .
$$

The power captured from the sea (hydrodynamic power) can also be defined by

$$
P_{a}=\omega_{r} T_{a} .
$$

Moreover,

$$
C_{q}(\lambda)=\frac{C_{p}(\lambda)}{\lambda} .
$$

From functions (2), (3), (4), and (5), we obtain

$$
T_{a}=\frac{1}{2} \pi \rho R^{3} C_{q}(\lambda) v^{2} .
$$

When the pitch angle $\beta$ is fixed, the water energy conversion system can only operate at some special $\lambda$ to capture the maximum power up to the rated speed. This special $\lambda$ is called the optimum tip speed ratio: $\lambda_{\text {opt }}$.

The optimum torque can be described by

$$
T_{\mathrm{opt}}=k_{\mathrm{opt}} \omega^{2},
$$

where the torque constant $k_{\text {opt }}$ is constant.

In the structural model in this paper, the pitch of the water turbines is manipulated using mechanical systems. Thus, the modified model considered here is a fixed-pitch turbine where $\beta=0$.

So we have

$$
k_{\mathrm{opt}}=\frac{1}{2 \lambda_{\mathrm{opt}}^{2}} \rho \pi R^{3} C_{q \max } .
$$

To study the kinetic properties of the generation system, the function of the power coefficient versus the tip speed ratio must be obtained. To obtain relatively accurate results for the rotating rotor, we first considered the hydrodynamic performance using the CFD method. Hence, two-dimensional numerical simulations were performed using the commercial code FLUENT 13.0. In this paper, we introduce a standard two-equation $k-\epsilon$ model to predict the turbulence effects in the transient predictions [25], which is widely used and able to simulate many flow regimes. The model is based on the transport equations for the turbulence kinetic energy $k$ and its dissipation rate $\epsilon$. The coefficients of torque and power are given by

$$
\begin{aligned}
C_{m} & =\frac{M}{0.5 \rho U^{2} R S}, \\
C_{p} & =\frac{P}{0.5 \rho U^{3} S}=\lambda C_{m},
\end{aligned}
$$

$$
S=2 R H .
$$

In these functions, $M$ is the blade torque, $S$ is the crosssectional area, and $H$ is the height of the blade. For 2D simulations, the unit height $H=1 \mathrm{~m}$ was used. 


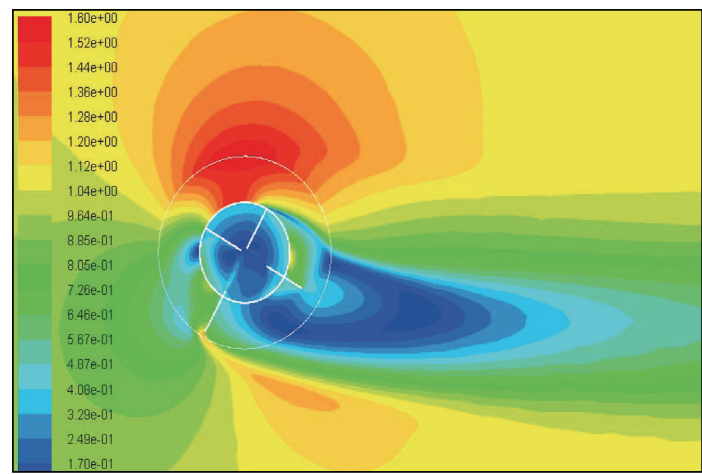

FIgURE 4: Velocity contours at a flow coefficient of $\lambda=0.4$ and a rotational angle of $\theta=60^{\circ}$.

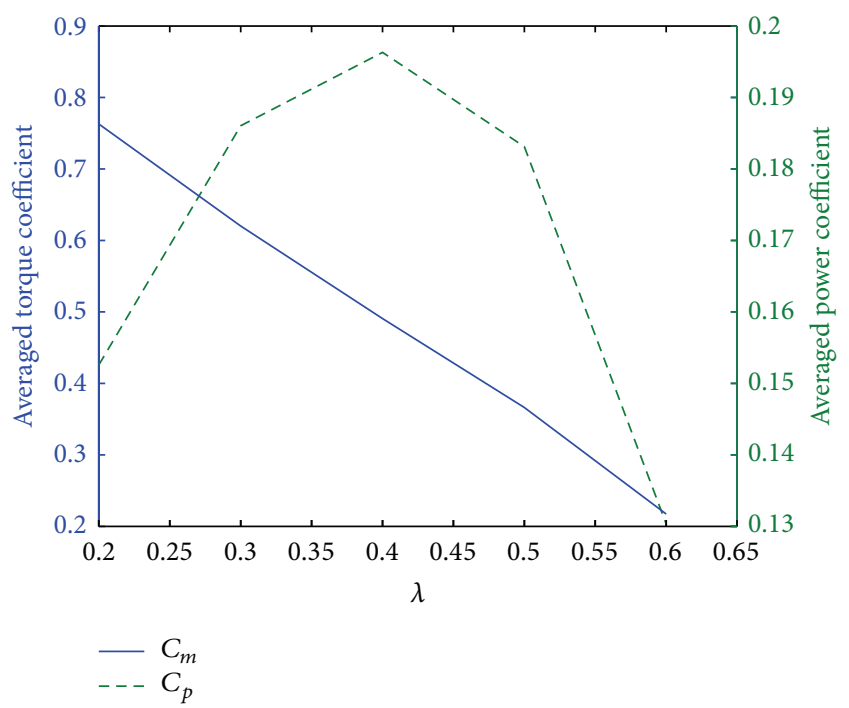

FIgURE 5: Coefficients of the averaged torque and power versus the flow coefficient.

To obtain the specific output performance of the turbine, several cases were investigated for rotor angular velocities between $0.4 \mathrm{~m} / \mathrm{s}$ and $1.2 \mathrm{~m} / \mathrm{s}$. Convergence is determined by the order of magnitude of the residuals. The drop of all scaled residuals below $10^{-5}$ is employed as the convergence criterion.

Figure 4 shows the contours of the absolute velocity in the flow field obtained at a flow coefficient of $\lambda=0.4$ and a rotational angle of $\theta=60^{\circ}$. Figure 5 indicates that velocity drop occurs when water passes the turbine, mainly caused by the advancing blade, which stretches to the outside of the drum and disturbs the flow. Because of the shielding of the drum, the flow inside the drum has a relatively small velocity and is less turbulent than that outside the drum. The flow velocity drops substantially when it passes the extended part of the blade, suggesting that the extended part of the blade contributes significantly to the total torque output, whereas the inside part of the blade hardly makes any contribution to the total torque.
TABle 2: Power coefficient $\left(C_{p}\right)$ versus tip speed ratio $\lambda$.

\begin{tabular}{cccccc}
\hline$\lambda$ & 0.2 & 0.3 & 0.4 & 0.5 & 0.6 \\
\hline$C_{p}$ & 0.15258 & 0.186044 & 0.1963 & 0.183111 & 0.130346 \\
\hline
\end{tabular}

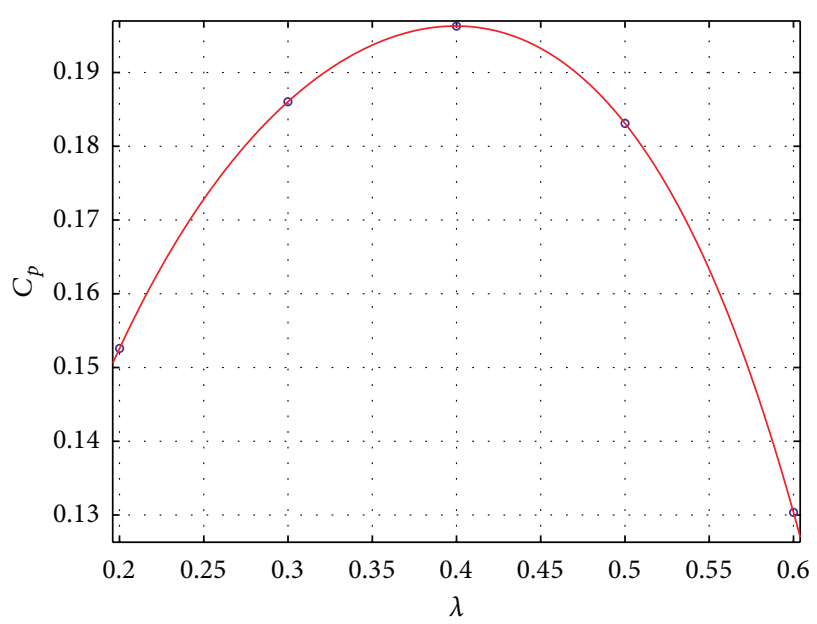

Figure 6: The power coefficient $C_{p}(\lambda)$ curve.

The CFD simulations are presented in Table 2, which lists the different values of the power coefficient $\left(C_{p}\right)$ as $\lambda$ varies.

The values in Table 2 are utilized to obtain the power coefficient $\left(C_{p}\right)$ versus the tip speed ratio curve using the least squares estimation method (see Figure 6). The quadratic fitting function is as follows:

$$
\begin{aligned}
C_{p}= & 7.125 \lambda^{5}-12.7396 \lambda^{3}+7.955 \lambda^{2}-1.0893 \lambda \\
& +0.1648 .
\end{aligned}
$$

For a specific water flow velocity, the averaged power coefficient increases with the flow coefficient until reaching the maximum point; subsequently, further increases in the flow coefficient lead to a decrease in the averaged power coefficient. The maximum averaged power coefficient is obtained at $\lambda=0.4$, with a value of 0.1963 , which is competitive with current drag-type turbines.

2.3. Hydrodynamic Model of the System. The VAT drive train dynamics are given in Figure 7. In Figure 7, the hydrodynamic torque $T_{a}$ will lead the VAT to operate at speed $\omega_{r}$. The lowspeed torque $T_{1 \mathrm{~s}}$ exerts a braking torque on the rotor. The generator is driven by the high-speed torque $T_{\mathrm{hs}}$ and braked by the generator electromagnetic torque $T_{g}$. The gearbox increases the rotor speed by the gearbox ratio $n_{g}$ to the generator speed $\omega_{g}$ and augments the low-speed torque.

The rotor dynamics equations are as follows:

$$
\begin{gathered}
J_{r} \dot{\omega}_{r}=T_{a}-K_{r} \omega_{r}-B_{r} \theta_{r}-T_{\mathrm{ls}}, \\
J_{g} \dot{\omega}_{g}=T_{\mathrm{hs}}-K_{g} \omega_{g}-B_{g} \theta_{g}-T_{g} .
\end{gathered}
$$




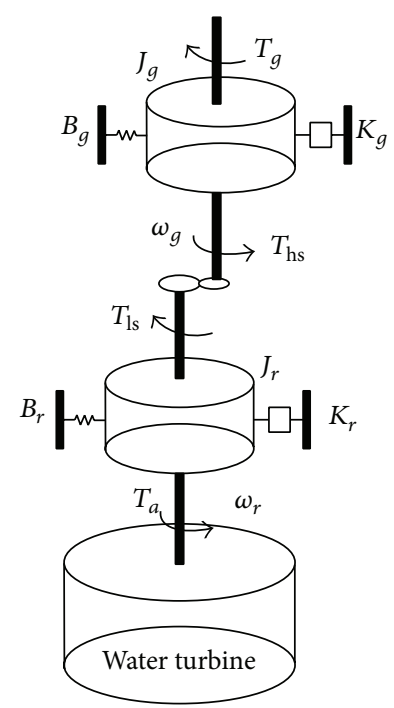

Figure 7: VAT drive train dynamics.

The gearbox ratio $n_{g}$ is defined as

$$
n_{g}=\frac{\omega_{g}}{\omega_{r}}=\frac{T_{\mathrm{ls}}}{T_{\mathrm{hs}}}
$$

After combining (11) and (12), we obtain

$$
J_{t} \dot{\omega}_{r}=T_{a}-K_{t} \omega_{r}-B_{t} \theta_{r}-T_{g}
$$

with

$$
\begin{gathered}
J_{t}=J_{r}+n_{g}^{2} J_{g}, \\
K_{t}=K_{r}+n_{g}^{2} K_{g}, \\
B_{t}=B_{r}+n_{g}^{2} B_{g} .
\end{gathered}
$$

In the equation above, the combined inertia of the rotor and the generator dominates, and the external stiffness $B_{t}$ is low enough to be neglected. We represent the drive train using a single lumped mass for control purposes, and the following simplified model is proposed:

$$
J_{t} \dot{\omega}_{r}=T_{a}-K_{t} \omega_{r}-T_{g} .
$$

We finally obtain the generated power by

$$
P_{g}=T_{g} \omega_{r} .
$$

Equation (10) indicates that the system is highly nonlinear in terms of the square wave speed and $C_{p}$ function. According to the linear model described in $[26,27]$, we performed modeling to determine the form used to represent optimal control as follows:

$$
\Delta x=x-x_{\mathrm{opt}} .
$$

Near the optimal point, we define the hydrodynamic torque variation by

$$
\Delta T_{a}=\gamma \Delta \omega_{r}+(2-\gamma) \Delta v
$$

with

$$
\begin{aligned}
\Delta T_{a} & =T_{a}-\overline{T_{a}}, \\
\Delta \omega_{r} & =\omega_{r}-\overline{\omega_{r}}, \\
\Delta v & =v-\bar{v} .
\end{aligned}
$$

The torque parameter $\gamma$ is defined as

$$
\gamma=\gamma\left(\lambda_{\mathrm{opt}}\right)=\frac{C_{p}\left(\lambda_{\mathrm{opt}}\right) \lambda_{\mathrm{opt}}-C_{p}\left(\lambda_{\mathrm{opt}}\right)}{C_{p}\left(\lambda_{\mathrm{opt}}\right)} .
$$

The wave speed model is given by

$$
\dot{v}(t)=-\frac{1}{T_{w}} v(t)+\frac{1}{T_{w}} w(t),
$$

where $T_{w}$ is the time constant of the filter and the turbulence component of the wave speed results from low-pass filtering of white noise $w(t)$.

After the linearization of (19), we have

$$
J_{T} \omega_{r}(t)=\Delta T_{a}-K_{t} \omega_{r}-\Delta T_{g}
$$

and the mechanical time constant $J_{T}=\left(\overline{\omega_{r}} / \overline{T_{a}}\right) J_{t}$. Finally, we can define the state space model as

$$
\dot{x}(t)=A x(t)+B u(t)+L e(t),
$$

where

$$
\begin{aligned}
A & =\left[\begin{array}{cc}
-\frac{K_{t}}{J_{T}} & \frac{1}{J_{T}} \\
\frac{\gamma}{T_{w}}-\frac{\gamma}{T_{w}} K_{t} & \frac{\lambda}{J_{T}}-\frac{1}{T_{w}}
\end{array}\right] ; \\
B & =\left[\begin{array}{c}
-\frac{1}{J_{T}} \\
-\frac{\gamma}{J_{T}}
\end{array}\right] ; \\
L & =\left[\begin{array}{c}
0 \\
\frac{2-\gamma}{T_{w}}
\end{array}\right], \\
x(t) & =\left[\begin{array}{l}
\Delta \omega_{r}(t) \\
\Delta T_{a}(t)
\end{array}\right], \\
u(t) & =\Delta T_{g} .
\end{aligned}
$$

The output $y(t)$ is defined as $y(t)=C x(t)$ when

$$
C=\left[\frac{2}{2-\gamma}-\frac{1}{2-\gamma}\right]^{T} .
$$

In this paper, the output is simplified as the variation in the rotor speed, and matrix $C$ is given when $\gamma=0$ by

$$
C=\left[\begin{array}{ll}
1 & -\frac{1}{2}
\end{array}\right]^{T} \text {. }
$$

The controller strategy is presented in Figure 8. 


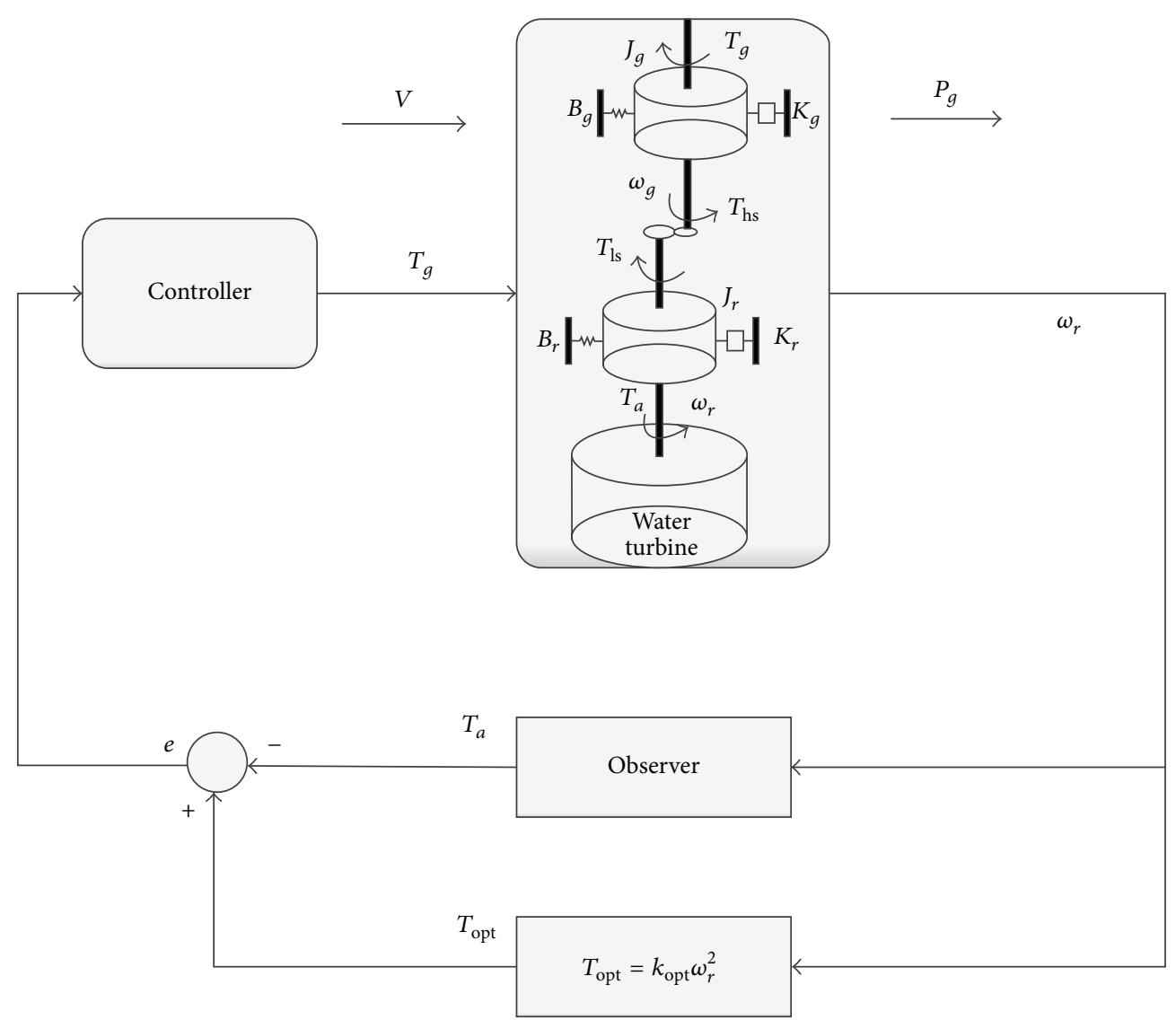

FIgURE 8: Control strategy.

2.4. Optimal LQT Control Description. Optimal control is a control concept that has been widely used for over 20 years, especially in the process industry [28-31]. With the development of optimization algorithms, researchers are now more interested in other areas of application. Optimal LQT control is a process model. Any process model capable of predicting future output signals based on initial values can be used to predict the future input within the prediction over the horizon time length. The predicted output signals are used to minimize the cost function based on the control purposes and applications. The dynamic equation and measured equation are given by

$$
\begin{aligned}
& X_{k+1}=A_{k} X_{k}+B_{k} U_{k}+W_{k}, \\
& Y_{k+1}=C_{k} X_{k} .
\end{aligned}
$$

The subscript $k$ indicates the dimension of each matrix. Another output is

$$
M_{k}=D_{k} X_{k}
$$

$C_{k}$ is required to track the instructed matrix $N_{k}$. One of the most popular cost functions uses the quadratic form for tracking references as follows:

$$
J=E\left\{\sum_{k=1}^{N} e_{k}^{T} \bar{Q}_{k} e_{k}+U_{k-1}^{T} \bar{R}_{k-1} U_{k}\right\},
$$

where

$$
e_{k}=N_{k}-M_{k}
$$

We assume that the instructed matrix $N_{k}$ is created by a known system, which is described by

$$
\begin{aligned}
Z_{k+1} & =E_{k+1} Z_{k}+F_{k} \xi_{k}, \\
N_{k} & =G_{k} Z_{k} .
\end{aligned}
$$

In the function above, $\xi_{k}$ is the white noise. To track the optimal output, we introduce the augmented state vector

$$
X_{k}^{a}=\left[\begin{array}{l}
X_{k} \\
Z_{k}
\end{array}\right]
$$

and augmented noise vector

$$
\xi_{k}^{a}=\left[\begin{array}{c}
W_{k} \\
\xi_{k}
\end{array}\right]
$$

The dynamic equation of $X_{k}^{a}$ then becomes

$$
X_{k+1}^{a}=A_{k+1}^{a} X_{k}+B_{k}^{a} U_{k}+F_{k}^{a} \xi^{a},
$$




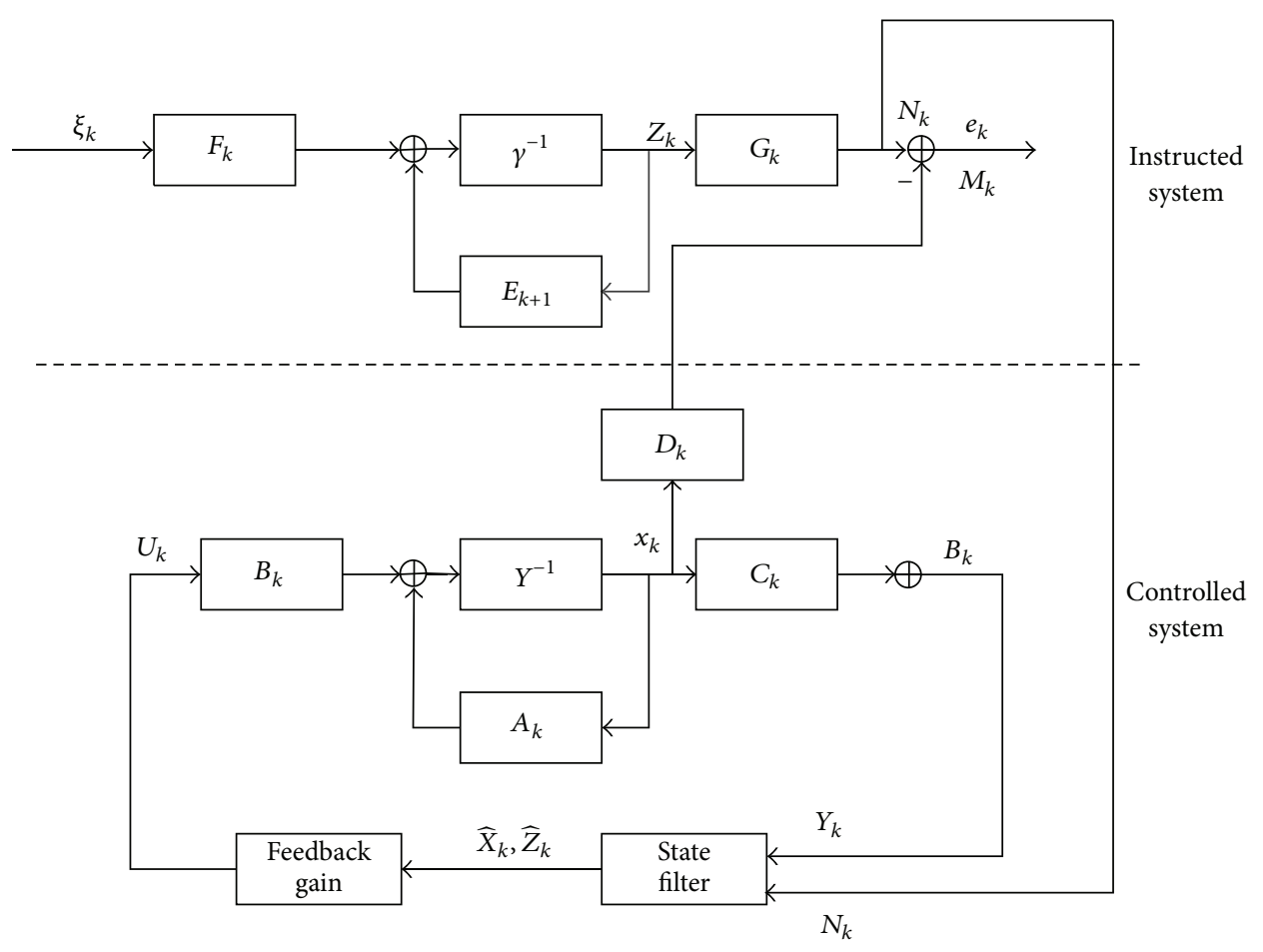

FIGURE 9: LQT controller schematic diagram.

where

$$
\begin{aligned}
A_{k+1}^{a} & =\left[\begin{array}{cc}
A_{k+1} & 0 \\
0 & E_{k+1}
\end{array}\right], \\
B_{k}^{a} & =\left[\begin{array}{c}
B_{k} \\
0
\end{array}\right], \\
F_{k}^{a} & =\left[\begin{array}{ll}
I & 0 \\
0 & F_{k}
\end{array}\right] .
\end{aligned}
$$

The updated output equation is

$$
Y_{k}^{a}=C_{k}^{a} X_{k}^{a}
$$

where

$$
\begin{aligned}
Y_{k}^{a} & =\left[\begin{array}{c}
Y_{k} \\
0
\end{array}\right], \\
C_{k}^{a} & =\left[\begin{array}{cc}
C_{k} & 0 \\
0 & G_{k}
\end{array}\right] .
\end{aligned}
$$

The index functions indicated by the augmented vector and augmented matrix are

$$
\begin{gathered}
e_{k}=N_{k}-M_{k}=G_{k} Z_{k}-D_{k} X_{k}=\left[-D_{k}, G_{k}\right] X_{k}^{a}, \\
J=E\left\{\sum_{k=1}^{N}\left[\left(X_{k}^{a}\right)^{T} Q_{k} X_{k}^{a}+U_{k-1}^{T} \bar{R}_{k-1} U_{k-1}\right]\right\}
\end{gathered}
$$

when

$$
Q_{k}=\left[-D_{k}, G_{k}\right]^{T} \bar{Q}_{k}\left[-D_{k}, G_{k}\right] .
$$

$X_{k}^{a}$ is a matrix consisting of $\widehat{X}_{k}$ and $\widehat{Z}_{k}$, which constitute the feedback loop. Figure 9 is the construct schematic diagram.

\section{Simulation Results and Discussion}

Because of the volatility of the wave speed, the hydrodynamic torque changes proportional to the rapid fluctuation of the wave speed. Therefore, the wave power conversion system is a time-varying parameter system. Formally, the wave speed is measured as accurately in this system as in others; however, the current simulation can measure the waves with errors of less than $10 \%$. The most widely used method is based on estimating the turbine shaft speed using Kalman filter. In our simulation, we describe the wave speed as a random signal with filtering, as shown in Figure 10, without considering large turbine effects. The wave velocity varies from $0.5 \mathrm{~m} / \mathrm{s}$ to $3.0 \mathrm{~m} / \mathrm{s}$, and the average wave maintains a velocity of $2.0 \mathrm{~m} / \mathrm{s}$ for $50 \mathrm{~s}$; this time interval is sufficient to simulate the seas' mild motion and observe the response of the controller.

The ocean power conversion system is designed to achieve two simple goals. Our primary goal was to capture the maximum wave power possible using the MPPT algorithm. Additionally, we aimed to determine how the system's load tolerance could be increased. To achieve the second objective, we minimized the rate of change of the generator torque control input by integrating the cost function (38). The control part we built in Simulink is presented in Figure 11. 


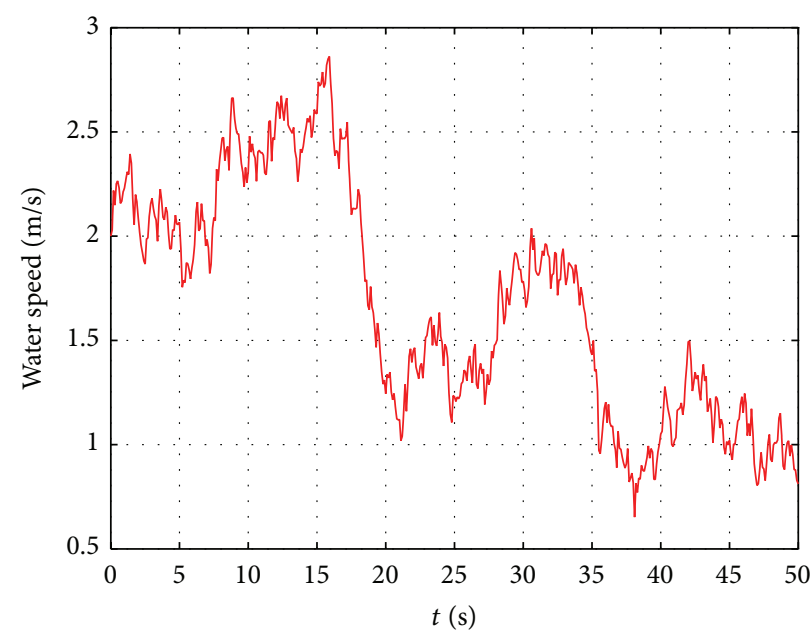

Figure 10: Water speed.

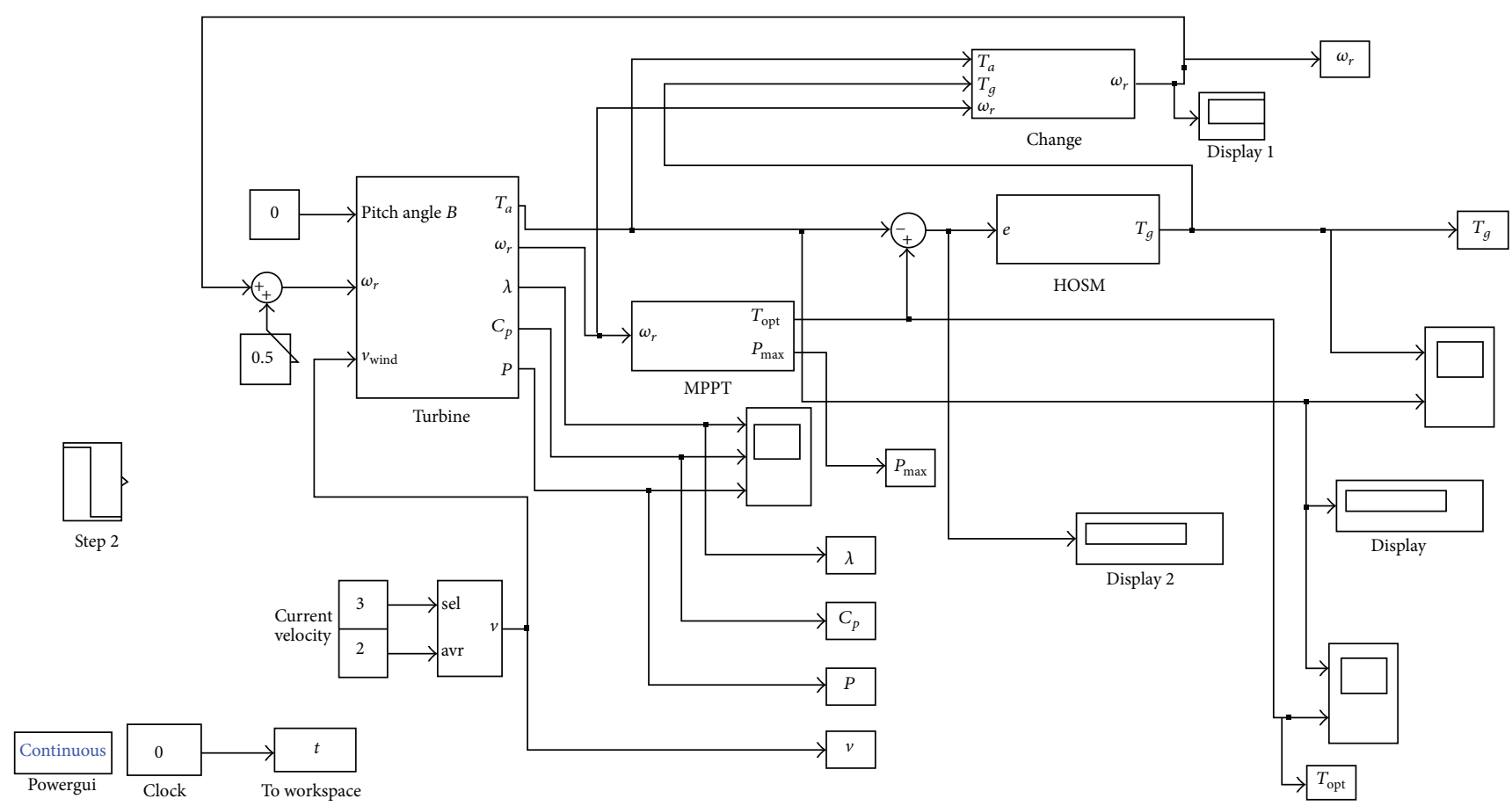

FIgUre 11: The Simulink model of the system.

In the paper, we adopted two controllers-a PID controller and an optimal controller-and compared them, verifying that the optimal LQT controller is capable of better tracing accuracy. The PID gains are $K_{P}=3, K_{I}=5$, and $K_{D}=0.05$. According to function (24), the subscript of dimension in optimal LQT controller is $k=2$. By comparing the errors between the actual and optimal torques, the following results were found. As expected, the wave's step changes substantially alter the optimal reference; the actual generator torque responses of the PID (blue) and optimal control (red) closely trace the optimal curve (black) in Figure 12(a). Thus, the optimal LQT controller provides better tracing stability and more accurate performance. We also plotted the absolute errors between the torque curves and the optimal curve in Figure 12(b) for additional clarity; again, the optimal controller clearly matches the ideal curve. Furthermore, the optimal LQT controller produces more accurate optimal values of the tip speed ratio, as shown in Figure 12(c).

The optimal LQT controller calculates the control input at each sampling time rather than simply producing the feedback gain once for each wave velocity. Figure 13 provides the output power and the maximum capture power. Figure 13(b) shows the small errors between the actual and optimal curves and the high efficiency of the optimal LQT control algorithm in maximizing power capture. 


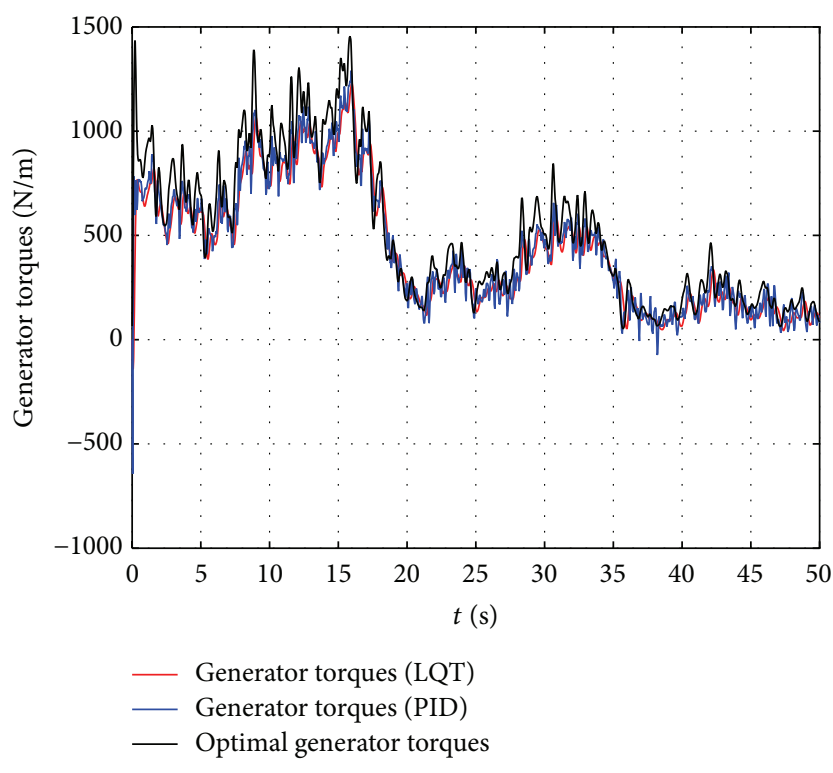

(a)

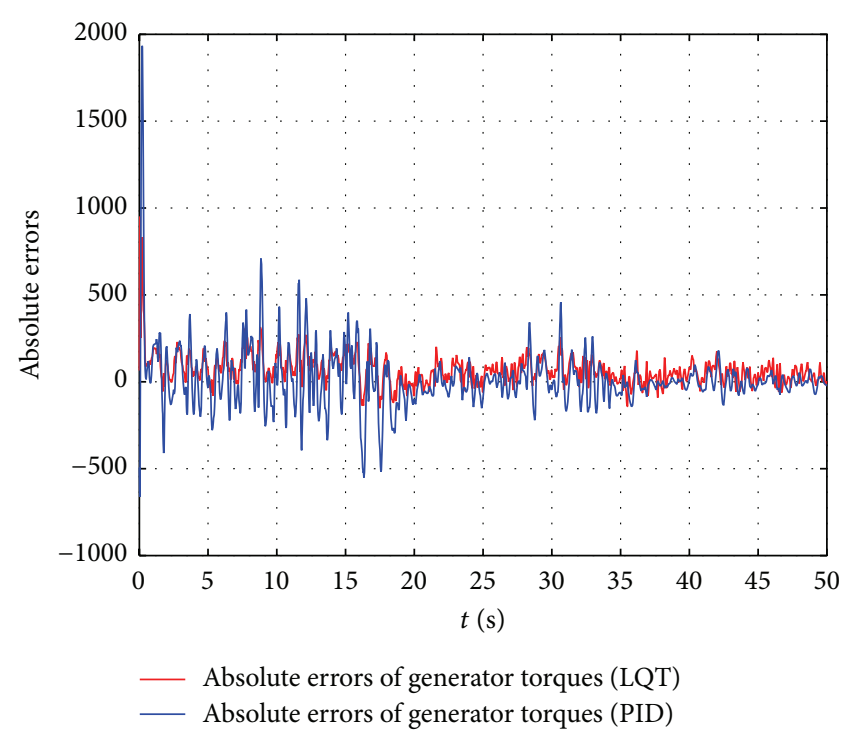

(b)

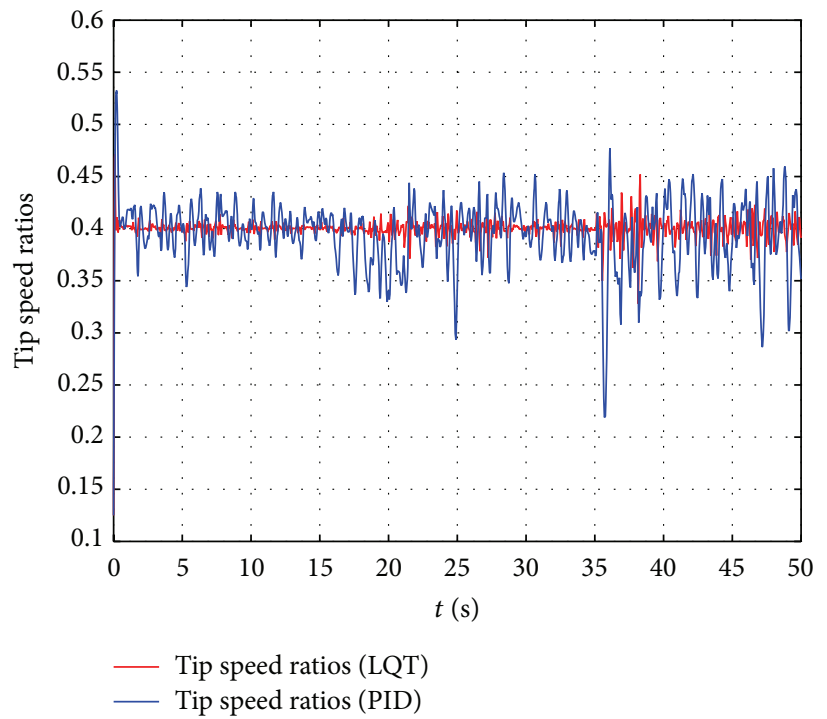

(c)

FIGURE 12: Wave profile responses: (a) actual generator torques, (b) the absolute error of the generator torques, and (c) tip speed ratios.

\section{Conclusions}

In this paper, we introduce a power generation control system for a new drag-type VAT with several retractable blades and describe how the turbine works under the repetitive wave disturbance model. To maximize power capture, we developed the model predictive controller using the linearized system to trace at the optimal tip speed ratio. Different wave speed types were investigated to solve the MPPT problem more efficiently. The simulation results show that the shaft follows the optimal curve perfectly, and, thus, the maximum power capture optimization occurs at convergence.

In the future, we will investigate other control algorithms and apply additional mathematical calculations to optimize their performance.

\section{Nomenclature}

$v: \quad$ Velocity of the water flow

$\rho: \quad$ Water density

$\lambda: \quad$ Tip speed ratio

$C_{p}(\lambda)$ : Power coefficient

$C_{q}(\lambda)$ : Torque coefficient

$\omega_{r}$ : Rotor speed

$\omega_{g}: \quad$ Generator speed

$T_{g}$ : Generator electromagnetic torque

$T_{\mathrm{ls}}$ : Low-speed torque

$T_{\mathrm{hs}}$ : High-speed torque

$K_{g}$ : Generator external damping

$K_{r}$ : $\quad$ Rotor external damping

$K_{t}$ : Turbine total external damping 


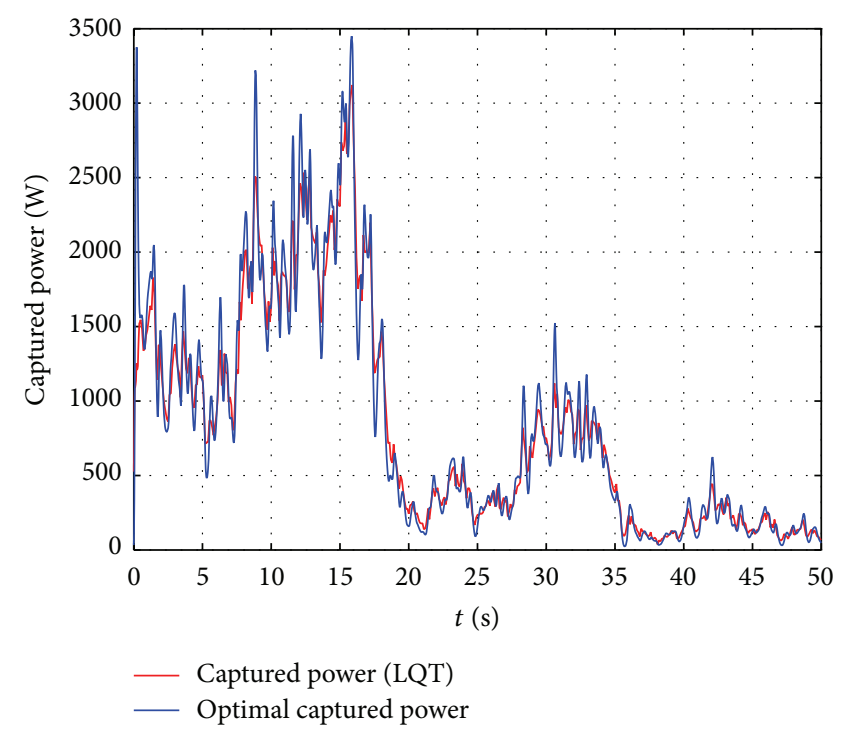

(a)

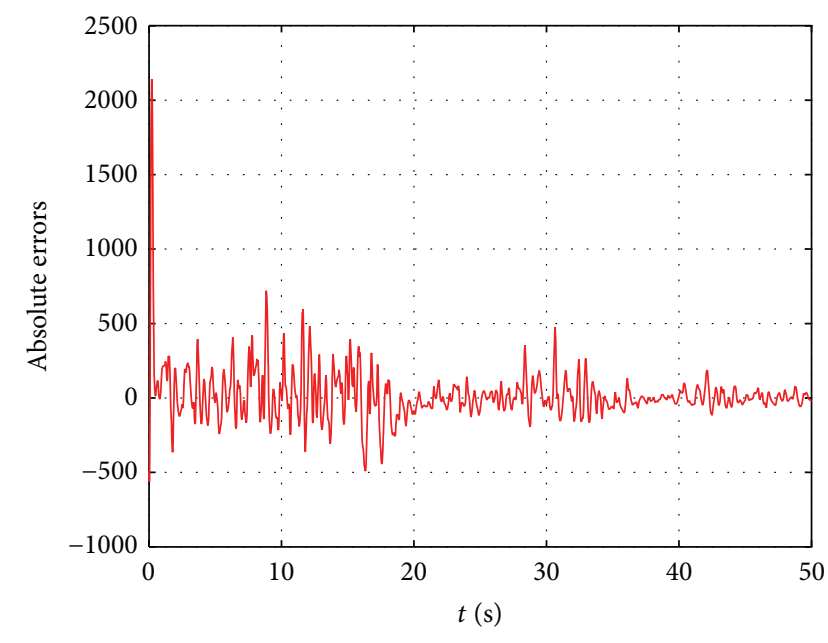

Absolute errors of captured power between actual and optimal

(b)

FIGURE 13: Captured power using optimal LQT control and the absolute errors.

$J_{r}:$ Rotor inertia

$J_{g}:$ Generator inertia

$J_{t}$ : Turbine total inertia

$B_{r}$ : Rotor external stiffness

$B_{g}$ : Generator external stiffness

$B_{t}$ : Turbine total external stiffness.

\section{Competing Interests}

The authors declare that no competing interests exist.

\section{Acknowledgments}

This research was supported by the National Natural Science Foundation of China (Grants no. 51179159 and no. 61572404).

\section{References}

[1] M. P. Kazmierkowski and M. Jasiński, "Power electronics for renewable sea wave energy," in Proceedings of the 12th International Conference on Optimization of Electrical and Electronic Equipment (OPTIM '10), pp. 4-9, IEEE, Basov, Romania, May 2010.

[2] B. Cahill and T. Lewis, "Wave energy resource characterization and the evaluation of potential wave farm sites," in Proceedings of the OCEANS 2011, pp. 1-10, IEEE, September 2011.

[3] F. Wu, X.-P. Zhang, P. Ju, and M. J. H. Sterling, "Modeling and control of AWS-based wave energy conversion system integrated into power grid," IEEE Transactions on Power Systems, vol. 23, no. 3, pp. 1196-1204, 2008.

[4] F. Fusco and J. V. Ringwood, "A simple and effective real-time controller for wave energy converters," IEEE Transactions on Sustainable Energy, vol. 4, no. 1, pp. 21-30, 2013.

[5] S. Lain and C. Osorio, "Simulation and evaluation of a straightbladed darrieus-type cross flow marine turbine," Journal of Scientific \& Industrial Research, vol. 69, no. 12, pp. 906-912, 2010.
[6] R. Howell, N. Qin, J. Edwards, and N. Durrani, "Wind tunnel and numerical study of a small vertical axis wind turbine," Renewable Energy, vol. 35, no. 2, pp. 412-422, 2010.

[7] K. Golecha, T. I. Eldho, and S. V. Prabhu, "Influence of the deflector plate on the performance of modified Savonius water turbine," Applied Energy, vol. 88, no. 9, pp. 3207-3217, 2011.

[8] B. D. Altan and M. Atilgan, "An experimental and numerical study on the improvement of the performance of Savonius wind rotor," Energy Conversion and Management, vol. 49, no. 12, pp. 3425-3432, 2008.

[9] K. Can, Z. Feng, and M. Xuejun, "Comparison study of a vertical-axis spiral rotor and a conventional savonius rotor," in Proceedings of the Asia-Pacific Power and Energy Engineering Conference (APPEEC '10), pp. 1-4, IEEE, Chengdu, China, March 2010.

[10] K. Pope, G. F. Naterer, I. Dincer, and E. Tsang, "Power correlation for vertical axis wind turbines with varying geometries," International Journal of Energy Research, vol. 35, no. 5, pp. 423435, 2011.

[11] Z. Mao and W. Tian, "Effect of the blade arc angle on the performance of a savonius wind turbine," Advances in Mechanical Engineering, vol. 7, no. 5, pp. 168-247, 2015.

[12] T. Wenlong, S. Baowei, M. Zhaoyong, and D. Hao, "Design of a novel vertical axis water turbine with retractable arc-type blades," Marine Technology Society Journal, vol. 47, no. 4, pp. 94100, 2013.

[13] R. Cui, Y. Li, and W. Yan, "Mutual information-based multiAUV path planning for scalar field sampling using multidimensional RRT*," IEEE Transactions on Systems, Man, and Cybernetics: Systems, 2016.

[14] J. Zhang, Z. Li, F. Xia, S. Tang, X. Shen, and B. Zhao, "Cooperative scheduling for adaptive duty cycling in asynchronous sensor networks," The Computer Journal, vol. 58, no. 6, pp. 12671279, 2015.

[15] R. Cui, J. Guo, and Z. Mao, "Adaptive backstepping control of wheeled inverted pendulums models," Nonlinear Dynamics, vol. 79, no. 1, pp. 501-511, 2015. 
[16] J. Zhang, M. Wang, and Z. Li, "Stochastic duty cycling for heterogenous energy harvesting networks," in Proceedings of the IEEE 34th International Performance Computing and Communications Conference (IPCCC '15), pp. 1-9, Nanjing, China, December 2015.

[17] W. He, S. S. Ge, B. V. E. How, Y. S. Choo, and K.-S. Hong, "Robust adaptive boundary control of a flexible marine riser with vessel dynamics," Automatica, vol. 47, no. 4, pp. 722-732, 2011.

[18] S. T. Pledgie, Y. Hao, A. M. Ferreira, S. K. Agrawal, and R. Murphey, "Groups of unmanned vehicles: differential flatness, trajectory planning, and control," in Proceedings of the IEEE International Conference on Robotics and Automation, vol. 4, pp. 3461-3466, May 2002.

[19] B. Kiumarsi, F. L. Lewis, H. Modares, A. Karimpour, and M.-B. Naghibi-Sistani, "Reinforcement q-learning for optimal tracking control of linear discrete-time systems with unknown dynamics," Automatica, vol. 50, no. 4, pp. 1167-1175, 2014.

[20] W. He, S. Zhang, and S. S. Ge, "Robust adaptive control of a thruster assisted position mooring system," Automatica, vol. 50, no. 7, pp. 1843-1851, 2014.

[21] K. H. Ang, G. Chong, and Y. Li, "PID control system analysis, design, and technology," IEEE Transactions on Control Systems Technology, vol. 13, no. 4, pp. 559-576, 2005.

[22] K. J. Åström and T. Hägglund, Advanced PID Control, ISA-The Instrumentation, Systems, and Automation Society, Research Triangle Park, NC, USA, 2006.

[23] D. Q. Dang, S. Wu, Y. Wang, and W. Cai, "Model predictive control for maximum power capture of variable speed wind turbines," in Proceedings of the International Power Electronics Conference (IPEC '10), pp. 274-279, IEEE, October 2010.

[24] B. Beltran, T. Ahmed-Ali, and M. E. H. Benbouzid, "High-order sliding-mode control of variable-speed wind turbines," IEEE Transactions on Industrial Electronics, vol. 56, no. 9, pp. 33143321, 2009.

[25] Ansys Fluent. 13.0 User's Guide, Ansys, Cecil Township, Pa, USA, 2010.

[26] Y. D. Song, B. Dhinakaran, and X. Y. Bao, "Variable speed control of wind turbines using nonlinear and adaptive algorithms," Journal of Wind Engineering and Industrial Aerodynamics, vol. 85, no. 3, pp. 293-308, 2000.

[27] D. Q. Dang, S. Wu, Y. Wang, and W. Cai, "Model predictive control for maximum power capture of variable speed wind turbines," in Proceedings of the 9th International Power and Energy Conference (IPEC '10), pp. 274-279, IEEE, Singapore, October 2010.

[28] H. Wang, Z. Chen, and Q. Jiang, "Optimal control method for wind farm to support temporary primary frequency control with minimised wind energy cost," IET Renewable Power Generation, vol. 9, no. 4, pp. 350-359, 2015.

[29] M. P. Schoen, J. Hals, and T. Moan, "Wave prediction and robust control of heaving wave energy devices for irregular waves," IEEE Transactions on Energy Conversion, vol. 26, no. 2, pp. 627638, 2011.

[30] M. S. Branicky, V. S. Borkar, and S. Mitter, "A unified framework for hybrid control: model and optimal control theory," IEEE Transactions on Automatic Control, vol. 43, no. 1, pp. 31-45, 1998.

[31] W. Tong, Wind Power Generation and Wind Turbine Design, Wit Press, 2010. 


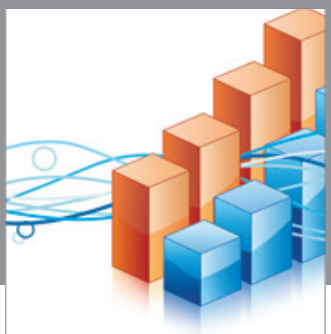

Advances in

Operations Research

vatem alat4

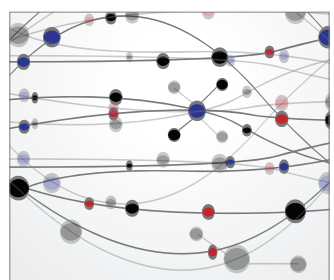

\section{The Scientific} World Journal
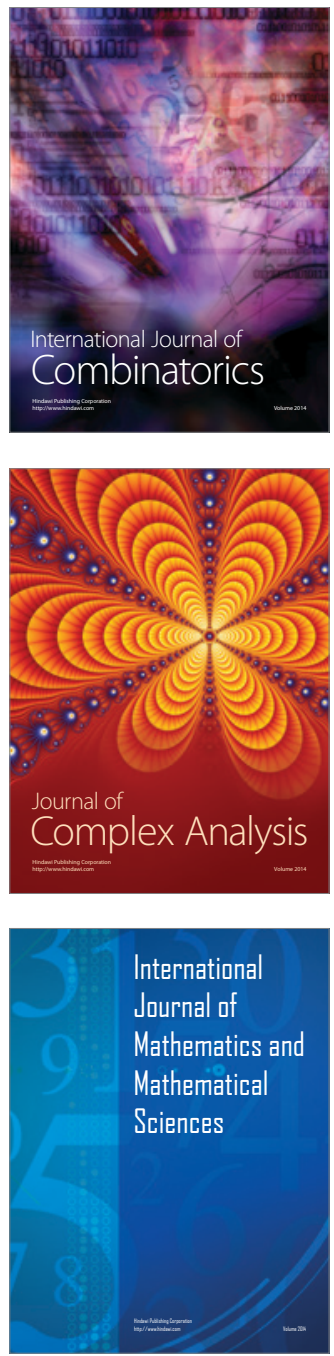
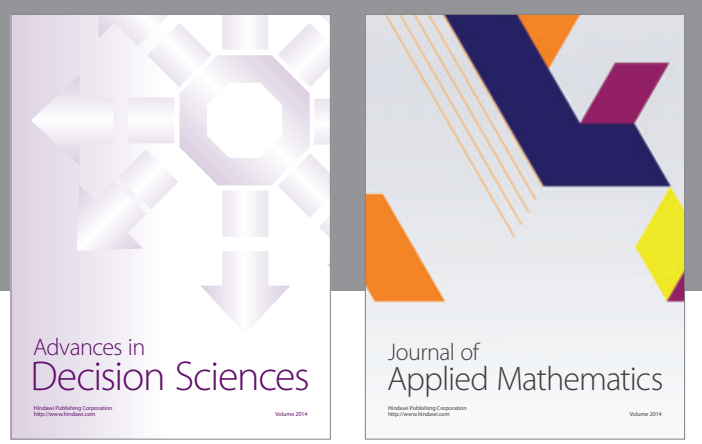

Algebra

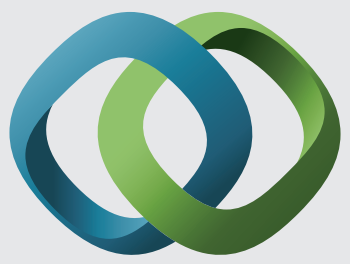

\section{Hindawi}

Submit your manuscripts at

http://www.hindawi.com
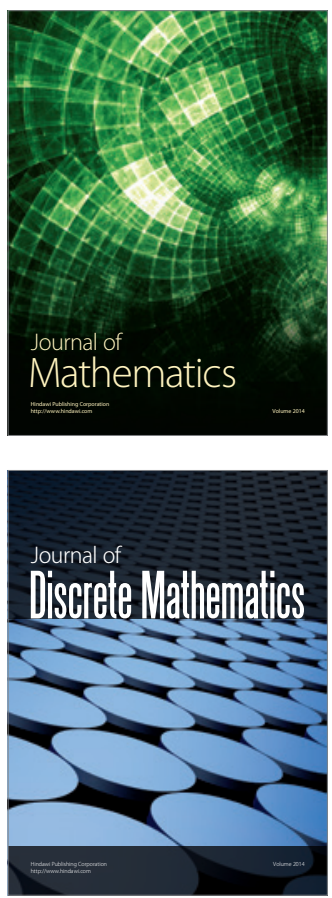

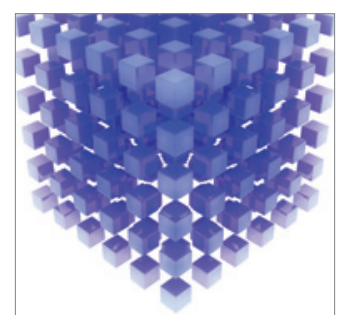

Mathematical Problems in Engineering
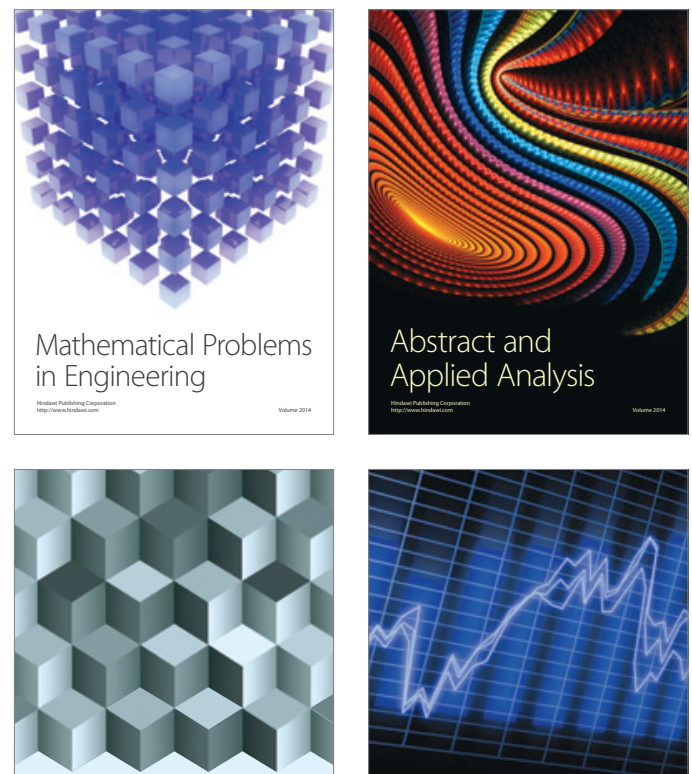

Journal of

Function Spaces

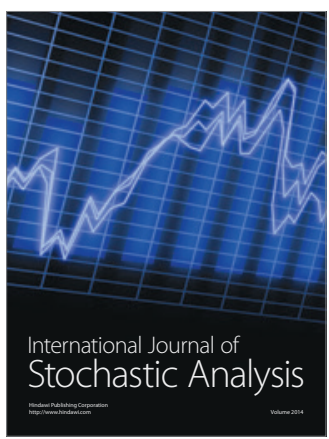

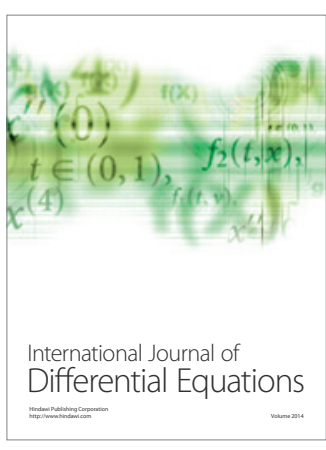
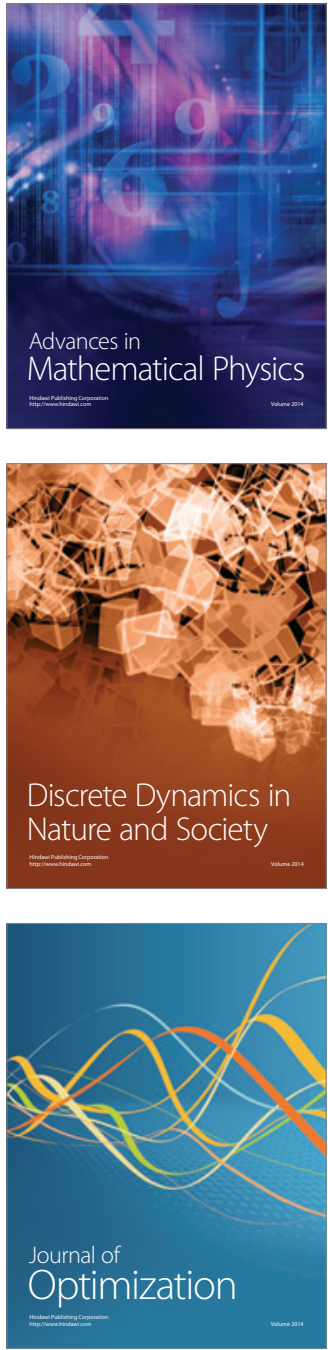